\title{
CUIDADOS NA ADMINISTRAÇÃO DE MEDICAMENTOS POR SONDA ENTERAL
}

Camila Montini de Almeida, Sandra Genaro

Universidade do Oeste Paulista - UNOESTE, Curso de Nutrição, Presidente Prudente, SP. e-mail: camilamontini@hotmail.com

\section{RESUMO}

O objetivo deste estudo foi discutir a utilização de medicamentos em pacientes submetidos à terapia nutricional enteral. $O$ estudo teve um delineamento observacional, transversal, de natureza quantitativa onde foram incluídos 30 pacientes, de ambos os sexos, maiores de 18 anos, internados no pronto socorro de um hospital público de uma cidade do interior paulista, que estavam em uso de Terapia Nutricional Enteral, com medicamentos prescritos pela sonda. Nos resultados foi verificado que do total de fármacos que foram utilizados, $42,85 \%$ apresentaram algum tipo de interação com os nutrientes da dieta, sendo $87,5 \%$ de interações negativas; $12,5 \%$ de interações positivas e $7,14 \%$ não foram encontrados nenhum tipo de interação. Concluindo, observou-se que há inúmeras situações que podem causar interações entre os fármacos prescritos e a dieta enteral, podendo levar a deficiências nutricionais e medicamentosas, diminuindo a eficácia de ambos, contribuindo para depleção do estado nutricional do paciente e dificultando sua recuperação.

Palavras-chave: Nutrição enteral, emergência, medicamentos, nutrientes, protocolo.

\section{CARE IN ENTERAL TUBE ADMINISTRATION}

\section{ABSTRACT}

The objective of this study was to discuss the use of drugs in patients submitted to enteral nutritional therapy. The study had a cross-sectional, observational design of a quantitative nature, in which 30 patients of both sexes, older than 18 years, were admitted to the emergency room of a public hospital in a city in the interior of São Paulo, who were using Nutritional Therapy Enteral, with drugs prescribed by the tube. In the results it was verified that of the total of drugs that were used, $42.85 \%$ presented some type of interaction with the nutrients of the diet, being $87.5 \%$ of negative interactions; $12.5 \%$ of positive interactions and $7.14 \%$ did not find any type of interaction. In conclusion, it was observed that there are numerous situations that can cause interactions between the prescribed drugs and the enteral diet, leading to nutritional and drug deficiencies, reducing the effectiveness of both, contributing to depletion of the patient's nutritional state and hindering its recovery.

Keywords: Enteral nutritional, emergency, medicines, nutrientes, protocol.

\section{INTRODUÇÃO}

A manutenção do organismo depende de nutrientes que são necessários e indispensáveis à nossa saúde. Alguns pacientes hospitalizados não possuem correta deglutição devido a sua condição clínica ou pela incapacidade de ingerir uma quantidade adequada de nutrientes para manter a saúde ou recuperar o estado nutricional, sendo necessária a introdução da terapia nutricional enteral ${ }^{1-3}$.

A terapia nutricional enteral (TNE) referese a um conjunto de procedimentos terapêuticos, que tem por objetivo, manter ou recuperar o estado nutricional do paciente por meio da 
nutrição enteral ${ }^{4}$, através da oferta de nutrientes para o trato gastrointestinal pelo uso de sondas ${ }^{5}$.

Apesar de vários avanços com a nutrição enteral, ainda é comum surgirem complicações que devem ser detectadas de forma precoce ${ }^{4}$. É importante ressaltar que os medicamentos de uso oral não são avaliados pelos fabricantes e agências reguladoras para o seu uso através da terapia nutricional enteral. Sabe-se que a administração incorreta de medicamento é capaz de obstruir a sonda pela formação de complexos insolúveis através da relação nutriçãomedicamento ou técnica inadequada de manipulação dos comprimidos introduzidos ${ }^{4,5}$.

As possíveis interações podem prejudicar a ação do medicamento e/ou alimento, alterando a necessidade do fármaco para o tratamento da doença, gerando aumento no custo e tempo de internação hospitalar, além de desnutrição pela má absorção dos nutrientes ofertados ${ }^{1}$.

Vários medicamentos são capazes de causar efeitos colaterais como náuseas, vômitos, diarreia, dor abdominal, repercutindo na qualidade da terapia nutricional. O método de administração da dieta enteral, de forma contínua, é considerado um fator complicador, pois requer a pausa da alimentação para administração de medicamentos interrompendo diversas vezes o processo de infusão da dieta ${ }^{6}$.

Diante do exposto, a realização deste trabalho teve como objetivo discutir a utilização de medicamentos em pacientes submetidos à terapia nutricional enteral.

\section{MÉTODOS}

O estudo teve delineamento
observacional, transversal, de natureza quantitativa. Foram incluídos 30 pacientes, de ambos os sexos, maiores de 18 anos, internados no pronto socorro de um hospital público de uma cidade do interior paulista no período de agosto a novembro de 2017, que estavam em uso de Terapia Nutricional Enteral (TNE), com medicamentos prescritos pela sonda.

O protocolo foi avaliado e aprovado do Comitê de Ética para Pesquisa sob o número CAAE 73918917.0.0000.5515, e os procedimentos adotados obedeceram aos Critérios da Ética em Pesquisa com Seres Humanos conforme Resolução no. 466/2012 do Conselho Nacional de Saúde.

Após a seleção dos pacientes com prescrição de TNE através da busca em prontuários, foi preenchido um questionário não estruturado, que informavam os dados de identificação do paciente, como idade, hipótese diagnóstica, tipo de medicamentos administrados, via de administração, posologia; dieta prescrita, via de acesso, modo de administração, intercorrências e características da dieta.

Para análise estatística, os dados foram organizados e foi elaborada uma planilha no programa EXCEL a qual era alimentada diariamente após cada coleta. Os dados quantitativos foram analisados por meio da estatística simples e descritiva e os valores apresentados em média \pm desvio padrão e valores percentuais.

\section{RESULTADOS}

Do total de pacientes avaliados, $43,33 \%$ eram do sexo feminino, com idade média de $74,76 \pm 16,47$ anos, e $56,66 \%$ do sexo masculino com idade média de 70,0 $\pm 16,36$ anos.

As doenças que mais se destacaram como motivos de internação estão descritas na Tabela 1.

Tabela 1. Principais motivos de internação.

\begin{tabular}{lccl}
\hline \multicolumn{1}{c}{ Sistemas } & $\mathbf{n}$ & $\mathbf{\%}$ & Principal motivo (\%) \\
\hline Nervoso & 7 & 23,3 & Acidente Vascular Encefálico (AVE): 100 \\
$\begin{array}{l}\text { Digestório e Anexos } \\
\text { Cardiorrespiratório }\end{array}$ & 9 & 30 & $\begin{array}{l}\text { Dor abdominal: } 75 \\
\text { Doença Pulmonar Obstrutiva Crônica (DPOC): } 33 \\
\text { Pneumonia: } 33\end{array}$ \\
Urinário & 20 & 13,3 & $\begin{array}{l}\text { Insuficiência Cardíaca: } 33 \\
\text { Insuficiência do Trato Urinário: } 50 \\
\text { Insuficiência Renal Aguda: } 50\end{array}$ \\
$\begin{array}{l}\text { Doença Autoimune } \\
\text { Neoplasias }\end{array}$ & 3 & 10 & $\begin{array}{l}\text { Doenças autoimunes inflamatórias: } 100 \\
\text { Neoplasia de Próstata: } 100\end{array}$ \\
\hline
\end{tabular}


A escolha do posicionamento da sonda depende de o paciente apresentar risco de aspiração ou intolerância, como distensão, refluxo ou gastroparesia ${ }^{7}$. Neste estudo, $96,7 \%$ dos pacientes tiveram posicionamento de sonda nasogástrica e 3,3\% tiveram posicionamento via gastrostomia.

Com relação aos métodos de administração, 70\% utilizavam sistema aberto, através de administração intermitente, em um intervalo de 4 horas. O restante (30\%) utilizava sistema fechado através de administração contínua da dieta, por bomba de infusão, com pausa de $2 \mathrm{~h}$ no período.

Através de observação no prontuário, foram identificados 56 tipos de diferentes de medicamentos, com média de 4,03 $\pm 2,18$ por paciente, o que corrobora com o estudo feito por Lopes, Carvalho e Freitas ${ }^{1}$ e Basso e Pinheiro ${ }^{4}$ que encontraram uma média de 1,36 e 7,8 medicamentos por paciente, respectivamente.

Os medicamentos mais utilizados foram agrupados segundo a classificação ATC
(Anatomical Therapeutic Cheical Code), utilizada internacionalmente para distribuir os fármacos em diferentes grupos e subgrupos de acordo com o órgão ou sistema sobre o qual atuam e segundo as suas propriedades químicas, farmacológicas e terapêuticas ${ }^{8,9}$. Conforme essa classificação foi observado que os medicamentos mais utilizados eram para tratamento de doenças do Sistema Nervoso (35,71\%), seguido do Sistema Cardiovascular $(33,92 \%)$ e Aparelho Digestório e Metabólico (17,85\%).

Do total de fármacos que foram utilizados, $42,85 \%$ apresentaram algum tipo de interação com os nutrientes da dieta, sendo $87,5 \%$ de interações negativas; $12,5 \%$ de interações positivas e $7,14 \%$ não foram encontrados nenhum tipo de interação.

O Quadro 1 exibe os medicamentos utilizados pelos pacientes, bem como seu efeito e interações positivas ou negativas com os nutrientes e o cateter da sonda.

Quadro 1. Relação de medicamentos, sua indicação, presença de interação.

\begin{tabular}{|c|c|c|c|c|}
\hline MEDICAMENTOS & EFEITO & INTE & AÇÃO & TIPO DE INTERAÇÃO \\
\hline & & SIM & NÃO & \\
\hline $\begin{array}{l}\text { Ácido acetilsalicílico } \\
\text { (AAS) }\end{array}$ & Analgésico, antitérmico & $x$ & & $\begin{array}{l}\text { Reduz absorção de vitamina C, K, B1, } \\
\text { ácido fólico } \\
\text { Carboidrato reduz tempo de absorção } \\
\text { Alimento reduz absorção }\end{array}$ \\
\hline Ácido fólico & Antianêmico & & $x$ & - \\
\hline Ácido valproico & Anticonvulsivante & $x$ & & $\begin{array}{c}\text { Alimento pode retardar absorção - não } \\
\text { significativo } \\
\text { Risco de aderência a sonda }\end{array}$ \\
\hline Amiodarona & Antiarrítmico & & $x$ & - \\
\hline Anlodipino & Anti-hipertensivo & & $x$ & - \\
\hline Atenolol & Anti-hipertensivo & $x$ & & $\begin{array}{l}\text { Alimento reduz absorção em } 20 \% \\
\text { Pausar dieta } 1 \text { hora antes }\end{array}$ \\
\hline Atensina & Anti-hipertensivo & $x$ & & $\begin{array}{l}\text { Alimento reduz absorção } \\
\text { Pausar a dieta antes }\end{array}$ \\
\hline Azitromicina & Antibiótico & $x$ & & $\begin{array}{l}\text { Alimento reduz biodisponibilidade } \\
\text { Pausar dieta } 1 \text { hora antes, administrar, } \\
\text { reiniciar } 2 \text { horas depois }\end{array}$ \\
\hline Biperideno & $\begin{array}{l}\text { Anticolinérgico - } \\
\text { Parkinson }\end{array}$ & & $x$ & - \\
\hline Bisoprolol & Anti-hipertensivo & & $\mathrm{X}$ & - \\
\hline Bromoprida & Antiemético & & $x$ & - \\
\hline Captopril & Anti-hipertensivo & $x$ & & $\begin{array}{l}\text { Alimento reduz de } 30 \text { a } 40 \% \text { a absorção } \\
\text { Pausa dieta } 1 \text { hora antes }\end{array}$ \\
\hline
\end{tabular}




\begin{tabular}{|c|c|c|c|c|}
\hline Carbamazepina & Anticonvulsivante & $x$ & & $\begin{array}{l}\text { Alimento reduz absorção e aumenta } \\
\text { aderência a sonda } \\
\text { Pausar dieta } 1 \text { hora antes, administrar, } \\
\text { reiniciar } 2 \text { horas depois }\end{array}$ \\
\hline Carvedilol & Anti-hipertensivo & & $x$ & - \\
\hline Clonazepam & $\begin{array}{l}\text { Distúrbios epilépticos, } \\
\text { distúrbios de ansiedade }\end{array}$ & & $x$ & - \\
\hline Clopidogrel & $\begin{array}{l}\text { Antiagregante } \\
\text { plaquetário }\end{array}$ & & $x$ & - \\
\hline Clorpromazina & Antipsicótico & & $\mathrm{x}$ & $\begin{array}{l}\text { Se administrar junto com dieta ocorre } \\
\text { risco de precipitação } \\
\text { Não há interações }\end{array}$ \\
\hline Complexo B & Vitamina & & $x$ & $\begin{array}{l}\text { Drágea não pode triturar, possui } \\
\text { excipientes que podem obstruir a sonda }\end{array}$ \\
\hline Dipirona & Analgésico, antitérmico & & $x$ & - \\
\hline Donepezila & $\begin{array}{l}\text { Anticolinérgico - } \\
\text { Alzheimer }\end{array}$ & & $x$ & $\begin{array}{l}\text { Comprimido não pode triturar perde } \\
\text { eficácia }\end{array}$ \\
\hline Enalapril & Anti-hipertensivo & & $x$ & - \\
\hline Fenitoína & Anticonvulsivante & $x$ & & $\begin{array}{c}\text { Interage com a dieta e reduz a } \\
\text { concentração plasmática em } 50-80 \% \\
\text { Pausar a dieta } 1 \text { horas antes, } \\
\text { administrar, reiniciar } 1 \text { hora depois }\end{array}$ \\
\hline Fenobarbital & Anticonvulsivante & $\mathrm{x}$ & & $\begin{array}{c}\text { Interação positiva } \\
\text { Alimento favorece absorção/ aumenta } \\
\text { biodisponibilidade do medicamento }\end{array}$ \\
\hline Furosemida & Diurético & $x$ & & $\begin{array}{l}\text { O alimento reduz absorção } \\
\text { Depletor de sódio } \\
\text { Interage com tiamina }\end{array}$ \\
\hline $\begin{array}{l}\text { Haldol/ } \\
\text { haloperidol }\end{array}$ & Distúrbios psicóticos & & $x$ & $\begin{array}{l}\text { Se administrar junto com dieta ocorre } \\
\text { risco de precipitação } \\
\text { Não há interações }\end{array}$ \\
\hline Hidralazina & Anti-hipertensivo & $x$ & & $\begin{array}{c}\text { Alimento reduz absorção/concentração } \\
\text { plasmática } \\
\text { Pausar dieta antes pelo maior tempo } \\
\text { possível }\end{array}$ \\
\hline Hidroclorotiazida & Diurético & $x$ & & $\begin{array}{l}\text { Alimento reduz absorção } \\
\text { Pausar a dieta antes }\end{array}$ \\
\hline $\begin{array}{l}\text { Isossorbida/ } \\
\text { monocordil }\end{array}$ & Distúrbios cardíacos & $x$ & & $\begin{array}{l}\text { Alimento interfere na absorção } \\
\text { Pausar dieta } 1 \text { hora antes }\end{array}$ \\
\hline Kcl xarope & Repositor de Potássio & $x$ & & $\begin{array}{l}\text { Interação física com a dieta } \\
\text { Risco de precipitação e obstrução da } \\
\text { sonda }\end{array}$ \\
\hline Lactulose & Laxante & $x$ & & $\begin{array}{l}\text { Incompatível com a dieta } \\
\text { Altera absorção de nutrientes e produz } \\
\text { precipitação }\end{array}$ \\
\hline Levofloxacino & Antibiótico & $x$ & & $\begin{array}{l}\text { Alimento reduz em } 25 \% \text { absorção, forma } \\
\text { complexos com minerais } \\
\text { Pausar dieta para } 1 \text { hora, administrar, } \\
\text { reiniciar } 2 \text { horas depois }\end{array}$ \\
\hline $\begin{array}{l}\text { Levomepromazina/ } \\
\text { neosine }\end{array}$ & Antipsicótico & - & - & Não há estudo \\
\hline Losartana & Anti-hipertensivo & & $X$ & - \\
\hline Varfarina Sódica & Anticoagulante & $x$ & & Dieta rica em vitamina $\mathrm{K}$ diminui a \\
\hline
\end{tabular}


anticoagulação e constituintes da dieta diminuem absorção

Pausar 1 hora antes, administrar, reiniciar 1 hora depois

\begin{tabular}{lcc}
\hline $\begin{array}{l}\text { Brometo de } \\
\text { Piridostigmina }\end{array}$ & $\begin{array}{c}\text { Inibidor de colinesterase } \\
- \text { Miastenia gravis }\end{array}$ \\
\hline Metadona & Analgésico e Sedativo \\
\hline Metoprolol & $\begin{array}{c}\text { Beta-bloqueador e Anti- } \\
\text { hipertensivo }\end{array}$ & Xaxante \\
\hline Óleo mineral & \\
\hline Omeprazol & $\begin{array}{c}\text { Inibidor da bomba de } \\
\text { próton - gastrite e } \\
\text { úlceras }\end{array}$ \\
& X
\end{tabular}

Prednisona $\quad$ Corticoide
$\begin{gathered}\text { Reduz absorção de vitaminas A, C, B6, } \\ \text { ácido fólico, Ca, K, P e Mg } \\ \text { Pausar a dieta antes }\end{gathered}$

\begin{tabular}{llll}
\hline Levodopa & Antiparkisoniano & $X$ & Dietas hiperproteicas e com vitamina B6
\end{tabular}
diminuem absorção

Pausar dieta 1 hora antes, administrar, reiniciar 2 horas depois

\begin{tabular}{lcl}
\hline Prometazina & Anti-histamínico & $\mathrm{X}$ \\
\hline Propanolol & Anti-hipertensivo & $\mathrm{X}$
\end{tabular}

X -

Risco de obstrução da sonda

Pausar dieta 2 horas antes

Reduz absorção de vitamina B12 e Fe

Cápsula não indicada

Os microgrânulos não podem ser triturados pois perde eficácia, abrir cápsula e dissolver microgrânulos em suco ácido e administrar imediatamente Alimento favorece absorção/ aumenta biodisponibilidade do medicamento

Ranitidina Antagonista de receptor $\quad X \quad$ Reduz absorção de vitamina B12
$\mathrm{H} 2$ - gastrite e úlceras Comprimido revestido quando macerado pode causar obstrução da sonda Pausar dieta antes para administrar

\begin{tabular}{|c|c|c|c|c|}
\hline Risperidona & Antipsicótico & & $X$ & - \\
\hline Rivastgmina & $\begin{array}{l}\text { Anticolinérgico - } \\
\text { Alzheimer }\end{array}$ & - & - & Não há estudo \\
\hline Selegilina & $\begin{array}{l}\text { Antidepressivo e } \\
\text { Antiparkinsoniano }\end{array}$ & & $x$ & - \\
\hline Metoprolol & Anti-hipertensivo & - & - & Não há estudo \\
\hline Sertralina & $\begin{array}{l}\text { Transtorno de ansiedade } \\
\text { e depressão }\end{array}$ & & $x$ & - \\
\hline $\begin{array}{l}\text { Dicloridrato de } \\
\text { pramipexol }\end{array}$ & Antiparksoniano & & $X$ & - \\
\hline Sinvastatina & Anti-hiperlipidemico & $\mathrm{X}$ & & nteração positiv \\
\hline
\end{tabular}

Alimento favorece absorção/ aumenta biodisponibilidade do medicamento

\begin{tabular}{|c|c|c|c|}
\hline Tiamina & Vitamina & $x$ & - \\
\hline Topiramato & Anticonvulsivante & $x$ & - \\
\hline Valsartana & Anti-hipertensivo & $x$ & - \\
\hline $\begin{array}{l}\text { Diosmina } \\
\text { Hesperidina }\end{array}$ & $\begin{array}{c}\text { Tratamento de } \\
\text { insuficiência venosa }\end{array}$ & - & Não há estudo \\
\hline
\end{tabular}


profunda

Vitamina D Vitamina

Fonte: ${ }^{1,4,5,10-19}$

$X$

As interações negativas encontradas entre drogas e nutrientes foram aquelas administradas para doenças do sistema digestório e metabólico, sistema cardiovascular, doenças dermatológicas, anti-infecciosas (uso sistêmico) e sistema nervoso. Os medicamentos mais utilizados foram Ácido Acetilsalićlico (AAS; $23,07 \%)$, Ranitidina $(23,07 \%)$, Omeprazol $(9,61 \%)$, Isossorbida/Monocordil (7,69\%), Captopril $(5,76 \%)$, Hidralazina (5,76\%) e Azitromicina (5,76\%).

Durante o estudo não foi possível observar de forma criteriosa o modo de administração dos medicamentos, bem como se houve ou não trituração de medicamentos contraindicados. Observou-se somente que os medicamentos que não possuíam forma líquida, eram triturados e diluídos para sua administração.

\section{DISCUSSÃO}

O envelhecimento mundial está ganhando espaço dentro da sociedade devido à diminuição das taxas de fertilidade e mortalidade populacional ${ }^{20}$. A projeção demográfica idosa vem se expandindo em um ritmo acelerado quando comparado com outros grupos. A pirâmide etária sofreu inversão em seus diferentes estágios de vida, o que evidencia aumento cada vez maior da longevidade habitacional, tanto em países desenvolvidos como em países em desenvolvimento ${ }^{21}$. Tal acontecimento vem elevando as projeções populacionais da terceira idade e a estimativa para essa parcela é de que em 2050, a população dominante seja de indivíduos acima de sessenta anos e no Brasil, espera-se que isso ocorra até $2030^{22,23}$.

Em um estudo realizado em uma Unidade de Terapia Intensiva, no Rio Grande do Sul, a maior prevalência era da população idosa em uso de dieta enteral ${ }^{3}$. Esses dados confirmam o que foi encontrado nesta pesquisa no qual os idosos em uso de dieta enteral prevaleceram sobre os demais pacientes internados.

O envelhecimento é denominado um processo normal, iniciado na concepção e interrompido com o óbito ${ }^{24}$. Observa-se que nesse período há uma perda gradativa da massa corpórea além de mudanças na maioria dos sistemas do organismo devido a doenças crônicas, incapacidades, fragilidade, depressão e polifarmácia $^{25}$, justificando o aumento dessa população em Unidades de Terapias Intensivas.

Os motivos fundamentais que podem levar o indivíduo idoso a utilizar sonda para alimentação estão relacionados às doenças comuns à idade e podem apresentar relação com a disfagia, como as doenças neurológicas (AVE) e pulmonares $^{26}$, principais causas de internação deste estudo (Tabela 1).

Em um estudo feito por Carvalho et al. ${ }^{5}$, foi observado que as doenças neurológicas, como AVE eram as principais causas de internação. Segundo Waitzberg ${ }^{27}$, a indicação de nutrição enteral é comum nesse tipo de enfermidade devido às sequelas provocadas pela própria doença. Corroborando também com um trabalho realizado em um hospital de Santa Catarina ${ }^{28}$ o qual verificou que a principal causa de internação foi relacionada às doenças neurológicas, tendo a disfagia como critério para uso de sonda enteral.

Além das doenças, a perda ponderal encontra-se associada às alterações sistêmicas encontradas no idoso, como diminuição da secreção salivar, apresentando prejuízo na mastigação; atrofia da mucosa gástrica e intestinal $\mathrm{l}^{29}$; hiporexia; saciedade precoce, dentre outros, o que justifica a indicação de terapia nutricional enteral ${ }^{30}$.

Com relação à via de acesso, observamos que a nasogástrica foi a mais utilizada, justificando seu uso por curto período, de acordo com a literatura ${ }^{31}$. Essa via apresenta maior semelhança com a fisiologia normal do sistema digestório, preservando sua integridade, evitando a translocação bacteriana e a atrofia da mucosa gástrica e intestinal ${ }^{32}$. Entretanto, quando há necessidade de um tempo maior, ou seja, por mais de seis semanas, a melhor via de acesso é através da gastrostomia, uma sondagem cirúrgica realizada diretamente no estômago. A vantagem da gastrostomia para esses pacientes refere-se ao conforto, permitindo maior mobilidade, além de não provocar interferências na respiração, nem riscos de infecções ${ }^{31,33,34}$.

O método de administração da dieta mais utilizado nesta pesquisa foi o sistema aberto. As 
dietas de sistema aberto são aquelas produzidas em áreas específicas para manuseio, onde os nutrientes industrializados estão na forma de pó ou líquido e são reconstituídos, misturados e/ou envasados em frascos para serem administrados. Uma das vantagens desse sistema é que há possibilidade de modular o volume, calorias, macro e micronutrientes de forma individual. No entanto, o risco de contaminação se torna maior pela manipulação realizada. Outra maneira de administrar a dieta enteral é através do sistema fechado, no qual são utilizadas dietas industrializadas na forma de líquidos estéreis, acondicionadas em frascos hermeticamente fechados, prontos para serem administrados. São livres de manipulação, o qual diminui o risco de contaminações e infeç̧ões para o paciente. Em contrapartida, essas dietas não podem ser moduladas, tendo sua composição padronizada pelos laboratórios de origem ${ }^{35,36}$.

Além de serem utilizadas para alimentação, as sondas de nutrição também são empregadas para a administração de drogas ${ }^{26}$, já que 0 paciente não consegue deglutir medicamentos que não possuem similaridade por via endovenosa. Nesse caso, são necessários cuidados extras para evitar que ocorram complicações, como obstrução da sonda, distúrbios gastrointestinais, interações entre medicamentos e nutrientes ${ }^{37}$.

A administração de medicamentos via sonda enteral pode causar algumas incompatibilidades físico-químicas, farmacêuticas, farmacológicas e/ou farmacocinéticas ${ }^{26,37}$, além de sub ou supra dosagem de alguns fármacos, e/ou redução da absorção de alguns nutrientes, devido à interação medicamento/nutriente ${ }^{32}$. Antes de ser decidida a administração de fármacos pela sonda, deve-se considerar o método de administração da nutrição enteral. Como podem ocorrer interações, medicamentos e dieta enteral não devem ser administrados concomitantemente. 0 ideal é que a dieta seja pausada por um determinado tempo e os medicamentos administrados nos intervalos das infusões. Quando a dieta é oferecida de forma contínua, deve ser interrompida a infusão e realizada uma pausa de quinze a vinte minutos para administração de medicamentos e assim liberar a alimentação $^{1,26,32}$. Posterior à essa pausa, a taxa de infusão deverá ser ajustada para que o paciente receba todo $\mathrm{o}$ aporte nutricional requerido a fim de evitar perda pondera ${ }^{38}$.
A interação entre drogas e nutrientes é muito comum em pacientes que utilizam sonda como via de alimentação e, na maioria das vezes, são administrados fármacos concomitantemente com alimentos, podendo levar à diminuição da absorção do medicamento ou aumento de sua toxicidade devido à maior absorção, além de serem responsáveis pela alteração na motilidade intestinal ${ }^{1,6}$, como observado no Quadro 1 , que apresenta os medicamentos que foram utilizados pelos pacientes do estudo, bem como suas interações.

Alguns medicamentos que não apresentam interações com nutrientes não podem ser triturados ou amassados devido a probabilidade da perda de sua eficácia, nem misturados à dieta pelo risco de precipitação da mesma, levando a grandes chances de obstrução da sonda enteral $\left.\right|^{1,4,5,10,11,13,39,40}$. Tais medicamentos, quando triturados, sofrem destruição de seus revestimentos, levando a uma liberação imediata e rápida do fármaco, o que pode causar toxicidade, potencialmente grave ao paciente, como foi relatado em um estudo realizado por Renovato; Carvalho; Rocha ${ }^{41}$, o qual observaram que medicamentos de liberação entérica que foram triturados, acarretaram efeitos tóxicos aos pacientes de um hospital de Dourados, MS. Essa situação, além de inadequada é corriqueira. Alguns estudos analisaram o padrão de prescrições de pacientes com sonda enteral e demonstraram uma tendência a se prescrever medicamentos na forma farmacêutica de comprimidos simples ${ }^{42}$. Outro estudo realizado por Silva et al. ${ }^{42}$, em uma unidade hospitalar no Rio de Janeiro, verificou que $15 \%$ dos medicamentos dispensados aos pacientes eram classificados como "não trituráveis" pela literatura.

É importante observar o modo de apresentação dos medicamentos para administrá-los de maneira adequada, pois quando um comprimido é triturado, seu revestimento pode ser capaz de obstruir a sonda levando a uma necessidade de troca da mesma, o que acarreta em aumento nos custos e desconforto ao paciente ${ }^{7,43}$, além de ocasionar uma interrupção do fornecimento da dieta, causando déficit nutricional ao paciente $e^{7,44}$.

A recomendação é de que os medicamentos que não possam ser triturados, sejam substituídos por outro modo de apresentação para serem utilizados, como em sua forma líquida ${ }^{38,42}$, endovenosa ou 
intramuscular. No entanto, foi verificado na literatura que se não houver possibilidade de troca da forma farmacêutica de alguns medicamentos, os comprimidos e cápsulas podem ser triturados e diluídos desde que sejam seguidos protocolos para este fim, respeitando inclusive as características físico químicas do princípio ativo do medicamento ${ }^{16-18,38,42,45}$

É importante também que se tenha cuidado com o intervalo entre a administração do fármaco e da dieta, pois podem afetar a absorção de ambos. Como foi exposto, alguns medicamentos devem ser administrados longe dos alimentos devido a sua interação negativa, como diminuição na capacidade de absorção da própria droga ou de nutrientes específicos, podendo causar carências nutricionais ${ }^{39}$. A utilização inadequada de medicamentos pela sonda pode levar a um déficit nutricional do paciente, interferindo na recuperação de sua saúde, sendo um agravante para maior permanência hospitalar e aumento nos custos da internação ${ }^{1,11}$.

Para que sejam reduzidos os efeitos das interações entre drogas e nutrientes, é recomendado o planejamento com padronização de horários para administração de medicamentos em consonância com os horários da dieta, atentando-se também às prescrições de múltiplos medicamentos com modo de administração da dieta, de maneira contínua, no qual é utilizado bomba de infusão, em que há necessidade de pausas constantes, interrompendo o fornecimento alimentar ${ }^{44}$.

\section{CONCLUSÃO}

Diante do exposto, observou-se que há inúmeras situações que podem causar interações entre os fármacos prescritos e a dieta enteral, podendo levar a deficiências nutricionais e medicamentosas, diminuindo a eficácia de ambos, contribuindo para depleção do estado nutricional do paciente e dificultando sua recuperação.

A formação de uma equipe multiprofissional atuante, tendo pelo menos um profissional das profissões médico, farmacêutico e nutricionista, é imprescindível para identificar interações entre fármaco/nutrientes. Essa equipe contribuirá para a melhora e bem-estar do paciente, assim como, a implantação de ações multidisciplinares como protocolos, manuais e treinamentos como educação permanente. Um manual é de suma importância, para que haja uma uniformização e sistematização na prática dos profissionais a fim de diminuir a ocorrência de interações entre medicamentos e alimentos.

\section{REFERÊNCIAS}

1. Lopes ME, Carvalho RBN, Freitas RM. Analysis of possible food/nutriente and drug interactions in hospitalized patients. Einstein. 201;8(3):298302. DOI: https://doi.org/10.1590/s167945082010ao1672

2. Fontana RM. Assistência Farmacêutica na administração de fármacos via sonda enteral: um estudo personalizado. Rev Especialize On-line IPOG. 2015;1(10):1-19.

3. Cervo AS, Magnago TSBS, Carollo JB, Chagas $B P$, Oliveira AS, Urbanetto JS. Adverse events related to the use of enteral nutritional therapy. Rev Gaúcha Enfer. 2014;35(2):53-9. DOI: https://doi.org/10.1590/19831447.2014.02.42396

4. Basso AP, Pinheiro MS. Avaliação dos medicamentos prescritos para pacientes submetidos à terapia nutricional enteral no CTI. Rev Bras Farm Hosp Serv Saúde. 2014;5(1):12-8.

5. Carvalho AMR, Oliveira DC, Neto JEH, Martins $\mathrm{BC}$, Vieira VMSF, Silva IMM. Análise da prescrição de pacientes utilizando sonda enteral em um hospital universitário do Ceará. Rev Bras Farm Hosp Serv Saúde. 2010;1(1):1-24.

6. Heldt T, Loss SH. Interação fármaco-nutriente em unidade de terapia intensiva: revisão da literatura e recomendações atuais. Rev Bras Ter Intensiva. 2013;25(2):162-7. DOI: https://doi.org/10.5935/0103-507X.20130028

7. Nunes ALB, Koterba E, Alves VGF, Abrahão V, Correia MITD. Terapia nutricional no paciente grave. Projeto diretrizes. 2011.

8. Código ATC - Anvisa. 2018. Acesso em: 8 jan $2019 . \quad$ Disponível em: http://www7.anvisa.gov.br/datavisa/Substancia/ ATC.htm/

9. WHOCC - Collaborating Centre for Drug Statistics Methodology. 2018. Acesso em: 8 jan $2019 . \quad$ Disponível em: https://www.whocc.no/atc/structure_and_princi ples/ 
10. Martins C, Moreira SM, Pierosan SR. Interações droga nutriente. 2.ed. São Paulo: Ed. Nutroclínica; 2003.

11. Hanauer GN. Interações entre fármacos $x$ nutrientes em um grupo de idosos do município de Humaitá-RS. 2009. Trabalho de conclusão de curso (Bacharel em nutrição), Universidade do Extremo Sul Catarinense, Rio Grande do Sul.

12. Nascimento MMG, Ribeiro AQ. Compilação de base de dados com recomendações para administração de medicamentos via sonda enteral. Rev Bras Farm Hosp Serv. 2010;1(1):124.

13. Farina LO, Poletto G. Interações entre antibióticos e nutrientes: uma revisão com enfoque na atenção à saúde. Visão acadêmica. 2010;11(1):91-8. DOI: http://dx.doi.org/10.5380/acd.v11i1.21359

14. Guia de Fármaco x Nutriente - Ebserch. 2018. Acesso em: 8 jan 2019. Disponível em: http://www.ebserh.gov.br/documents/220250/1 293244/GUIA+F\%C3\%81RMACO+X+NUTRIENTE+ EM+CONSTRU\%C3\%87\%C3\%830.pdf/ebe6b0bd321d-4983-bb61-89c33d22f46e

15. Recomendações para administração de medicamentos via sonda - Ebserch. 2017. Acesso em: 8 jan 2019. Disponível em: http://www.ebserh.gov.br/documents/16692/24 53147/Anexo+Resolu\%C3\%A7\%C3\%A30+82++Guia+para+administra\%C3\%A7\%C3\%A30+de+m edicamentos+via+sonda.pdf/73f77f30-5bea49d8-82ba-9915284126bb

16. Ácido Acetilsalicílico: comprimidos. Responsável técnico Dr. Ronoel Caza de Dio. Manaus - AM. Fabricado por: Novamed Fabricação De Produtos Farmacêuticos Ltda. 2015. Bula de remédio.

17. Hoefler R. Ácido acetilsalicílico como antiagregante plaquetário: qual a conduta ideal? Boletim farmacoterapêutico. 2004;IX(4).

18. Moura MRL, Reyes FGR. Interação fármaconutriente: uma revisão. Rev Nutr. 2002;15(1):223-8. DOI: https://doi.org/10.1590/S141552732002000200011
19. Renovato RD, Carvalho PD, Rocha RSA. Investigação da técnica de administração de medicamentos por sondas enterais em hospital geral. Rev Enferm UERJ. 2010;18(2):173-8.

20. Nações Unidas no Brasil. A ONU e as pessoas idosas. 2017. Acesso em: 8 jan 2019. Disponível em: www.nacoesunidas.org

21. Anais Economia da longevidade: uma revisão da bibliografia brasileira sobre 0 envelhecimento populacional em Encontro da Associação Brasileira de Economia da Saúde 8. São Paulo: PUC/SP; 2007. Acesso em: 8 jan $2019 . \quad$ Disponível em: http://www.observatorionacionaldoidoso.fiocr uz.br/biblioteca/ artigos/45.pdf

22. Fechine BRA, Trompieri N. O processo de envelhecimento: as principais alterações que acontecem com o idoso com o passar dos anos. Rev Cient Int. 2012;20(1):106-32. DOI: https://doi.org/10.6020/1679-9844/2007

23. Reis C, Barbosa LML, Pimentel VP. O desafio do envelhecimento populacional na perspectiva sistêmica da saúde. BNDES Setorial. 2016:87124. Acesso em: 8 jan 2019. Disponível em: DOI:

https://web.bndes.gov.br/bib/jspui/handle/140 $\underline{8 / 9955}$

24. Harris NG. Nutrição no Envelhecimento. In: Mahan LK, Escott-Stump S, Raymond JL. Krause: alimentos, nutrição e dietoterapia. 13.ed. Rio de Janeiro: Elsevier; 2012. p.304-23.

25. Wellman NS, Kamp BJ. Nutrição e Envelhecimento. In: Mahan LK, Escott-Stump S, Raymond JL. Krause: alimentos, nutrição e dietoterapia. 13.ed. Rio de Janeiro: Elsevier; 2012. p.442-56.

26. Philippsen EB. Uso de terapia nutricional enteral via sonda em pacientes hospitalizados. Rev Especialize On-line IPOG. 2015;1(10):1-16

27. Waitzberg DL. Nutrição oral, enteral e parenteral na prática clínica. 3.ed. São Paulo: Atheneu; 2000. p.1167-75.

28. Campanella LCA, Silveira BM, Neto OR, Silva AA. Terapia nutricional enteral: a dieta prescrita é 
realmente infundida? Rev Bras Nutr Clin. 2008;23(1):21-5.

29. Scherer R, Scherer F, Conde SR, Bosco SMD. Estado nutricional e prevalência de doenças crônicas em idosos de um município do interior do Rio Grande do Sul. Rev Bras Geriatr Gerontol. 2013;16(4):769-79.

DOI: https://doi.org/10.1590/S1809$\underline{98232013000400011}$

30. Ribeiro C M. Medicamentos administrados por sonda nasoenteral em pacientes geriátricos de hospital terciário universitário. [Monografia]. Programa de Aprimoramento Profissional em Farmácia Hospitalar do Hospital das Clínicas da Faculdade de Medicina de Ribeirão Preto. 2013

31. Cunha SFC, Cômodo ARO, Silva FAA, Tomaz BA, Ribas DF, Marchini JS. Terapia nutrológica oral e enteral em pacientes com risco nutricional. Projeto Diretrizes. 2008.

32. Araujo APV. Estudo da utilização de medicamentos administrados por sonda nasoenteral e nasogástrica em um hospital terciário do distrito federal. [Monografia]. Curso de Farmácia, Universidade de Brasília, Faculdade de Ceilândia. 2014.

33. Santos JZ, Kemp R, Sankarankutty AK, Junior WS, Tirapelli LF, Silva Junior OC. Gastrostomia e jejunostomia: aspectos da evolução técnica e da ampliação das indicações. Simpósio Fundamentos em Clínica Cirúrgica - 3a Parte. Ribeirão Preto. 2011;44(1):39-50.

DOI:

https://doi.org/10.11606/issn.2176-

7262.v44i1p39-50

34. Catafesta J. Frequência de eventos adversos gastroenterologicos em pacientes com terapia nutricional enteral no hospital de clinicas de Porto Alegra (HCPA). [Monografia]. Programa de pós-graduação em medicina: ciências em gastroenterologia. Faculdade de Medicina do Rio Grande do Sul. 2010.

35. Silva SMR, Assis MCS, Silveira CRM, Beghetto MG, Mello ED. Open versus closed enteral nutrition systems for critically ill adults: is there a difference? Rev Assoc Med Bras. 2011;58(2):22933. DOI: https://doi.org/10.1016/S01044230(12)70185-9
36. Costa MF. Nutrição enteral: sistema aberto ou sistema fechado? Uma comparação de custo benefício. Rev Bras Nutr Clin. 2014;29(1):14-9.

37. Rodrigues JB, Martins FJ, Raposo NRB, Chicourel EL. Perfil de utilização de medicamentos por sonda enteral em pacientes de um hospital universitário. Rev Bras Farm Serv Saúde. 2014;5(3):23-7.

38. Hoefler R, Vidal JS. Administração de medicamentos por sonda. Boletim farmacoterapêutico. 2009; XIV(3 e 4).

39. Peixoto JS, Salci MA, Radovanovic CAT, Salci TP, Torres MM, Carreira L. Riscos da interação droga-nutriente em idosos de instituição de longa permanência. Rev Gaúcha Enferm. 2012;33(3): 156-64. DOI: https://doi.org/10.1590/S198314472012000300021

40. Reis AMM, Carvalho REFL, Faria LMP, Oliveira RC, Zago KSA, Cavelagna MF, Silva AG, Luis Neto $M$, Cassiani SHB. Prevalência e significância clínica de interações fármaco-nutrição enteral em unidades de terapia intensiva. Rev Bras Enferm. 2014;67(1):85-90.

41. Schutz V, Silva LD, Praça BFM, Santos MER. Revisão sobre a interação entre fármacos e nutrição enteral. Enfermería Global. 2011;22:110. DOI: https://doi.org/10.4321/S169561412011000200021

42. Silva LD, Lisboa CD. Consequências da interação entre nutrição enteral e fármacos administrados por sondas: uma revisão integrativa. Cogitare Enferm. 2011;16(1):134-40. DOI: $\quad$ https://doi.org/10.5380/ce.v16i1.21124

43. Gorzoni ML, Torre AD, Pires SL. Medicamentos e sondas de nutrição. Rev Assoc Med Bras. 2010;56(1):17-21. DOI: https://doi.org/10.1590/S010442302010000100009

44. Balbino Junior M. Manual: Medicamento oral x sonda. São Paulo: Serviço de Farmácia, Hospital Santa Rita; 2013.

45. Interação droga (fármaco) x nutriente - CRN 2. 2018. Acesso em: 8 jan 2019. Disponível em: http://crn2.org.br/crn2/conteudo/conteudo/sb/d roganutriente.pdf 